\title{
Indoor multipath effect study on the Locata system
}

Article in Journal of Applied Geodesy · November 2010

DOI: $10.1515 /$ jag.2010.012

CITATIONS

3

3 authors:

Lukasz Kosma Bonenberg

University of Nottingham

24 PUBLICATIONS 68 CITATIONS

SEE PROFILE

\section{Gethin Wyn Roberts}

University of the Faroe Islands

133 PUBLICATIONS 1,239 CITATIONS

SEE PROFILE
READS

109

\section{Craig Hancock}

University of Nottingham, Ningbo Campus 49 PUBLICATIONS 174 CITATIONS

SEE PROFILE

Some of the authors of this publication are also working on these related projects: 


\title{
Indoor multipath effect study on the Locata system
}

\author{
Lukasz K. Bonenberg, Craig M. Hancock and Gethin W. Roberts
}

\begin{abstract}
GNSS has become one of the most widespread measurement technologies, allowing $\mathrm{cm}$-level positioning accuracy using RTK or Network RTK. Unfortunately, the system's major drawbacks are the requirement for a clear view of the sky and accuracy dependent on the geometric distribution of the satellites, not only varying throughout the day but also prone to location specific problems. With widespread utilisation of GNSS for monitoring of manmade structures and other civil engineering tasks, such shortcomings can be critical.
\end{abstract}

One of possible solution is the deployment of a supporting system, such as Locata - a terrestrial positioning technology, which mitigates the need for a clear view of the sky and provides system integrity control.

This paper, part of the proposed integration feasibility study, presents Locata performance indoors, its capacity and mitigation methods.

Keywords. Locatalite, pseudolite, indoors, precise positioning, deformation monitoring.

\section{Introduction}

GNSS, with its low maintenance, cm level accuracy and convenience of use, is used extensively in the monitoring of manmade structures and in other civil engineering activities. Satellite positioning is heavily dependent on the number and geometric distribution of visible satellites. This weakness makes GNSS less reliable in areas with a limited view of the sky, such as urban canyons. Research (Roberts et al. 2006) has shown accuracy degradation in the North South direction in the UK and similar latitudes, due to the design of the GPS constellation. It can be expected that similar problems can occur worldwide. One of the solutions to such a problem is the implementation of a supporting system.

\subsection{Locata}

Locata is a terrestrial positioning technology, developed by the Locata Corporation and based on the pseudolite concept. Major advantages over the previous pseudolite designs are:
- Dual frequency signal (S1\&S6) within $2.4 \mathrm{GHz}$ license free ISM Band, preventing interference with GNSS signal.

- Digital signal and Direct Sequence Code Division Multiple Access (DS-CDMA) to combat near-far effect, noise and interference.

- Two spatially separated transmitting antennas.

- Clustering of signals $(2 \times 2)$ to detect and mitigate noise (multipath).

- TimeLoc procedure to precisely synchronise the network (LocataNet) (Montillet et al. 2009).

Locatalites position in a GNSS-like fashion, calculating position and clock corrections. This requires visibility of at least four Locatalites for successful 3D trilateration. As the Locata 'satellites' are relatively close to the receiver compared to GNSS satellites the signal strength is much stronger. Its tranreceivers are usually nearly coplanar, due to environmental restrictions, so obtaining accurate vertical coordinates is difficult. As with any terrestrial positioning system, Locata is highly susceptible to fading multipath effects.

\subsection{Integrated geometry}

Research at the University of Nottingham (Montillet et al. 2009) and the University of New South Wales (Barnes et al. 2007) indicate Locata as an alternative to GNSS in areas difficult for satellite positioning such as urban environments. The results indicated that the Locata system is able to position to centimetre level using carrier phase solutions.

On its own, any ground based positioning system struggles, with vertical component determination, due to environmental restrictions. Research into pseudolites (Meng et al. 2003, Yang et al. 2010) suggests that integration with GNSS elevates this and improves 3D accuracy considerably. Additionally, the static nature of Locatalites strengthens the solution, by providing predictable observations.

Compare observed $24 \mathrm{~h}$ long Horizontal Dilution of Precision (HDOP) (Leick 2004) values from Locata and simulated GPS HDOP for the same time span (Figure 1).

Locata's solution is very stable (DOP 1.1) while GPS's altering between 0.8 and 2.0, due to changing 


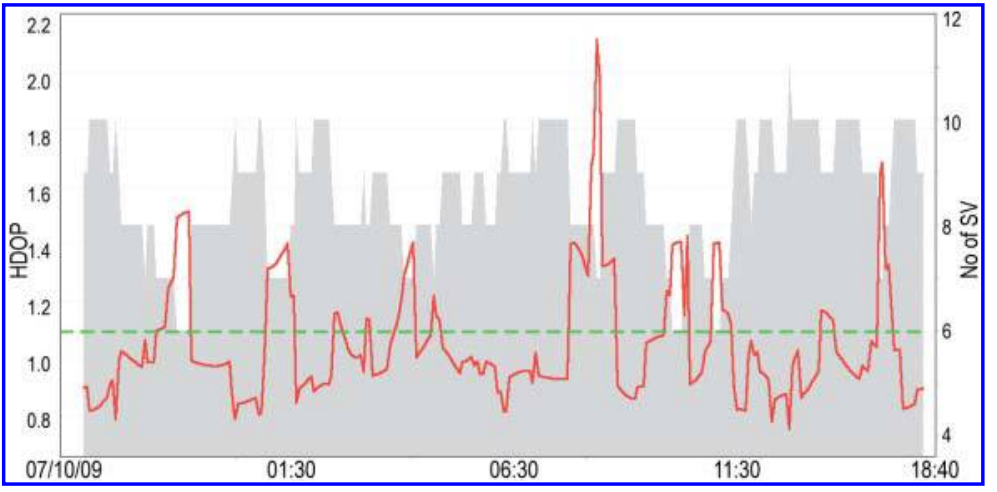

Figure 1: 24 h HDOP value for GPS (continuous) and Locata (dashed). geometric distribution of the satellites. Therefore static geometry can be utilised for GNSS integrity controls, especially in the strategic areas such as airports and harbours (Lee et al. 2008).

Unfortunately, any integration will be limited to Locata component in an indoor environment. This paper presents results from Locata indoor tests, conducted at the University of Nottingham, investigating the effects of multipath on the Locata signal and ways of mitigating it.

In order to create a baseline test for comparison purpose results from 3 different environments are analysed. Two outdoors (in Australia and UK) are discussed in Section 2, while Section 3 concentrates on the analysis of the indoor tests.

\section{Static tests}

\subsection{Problem description}

One of the problems facing any transmitter based system is noise. In the case of the terrestrial systems the major contributors are interference and multipath.

Interference in general lowers signal strength. Multipath, a non-direct signal, reduces power and shifts phase (Leick 2004, Yang et al. 2010). In extreme cases, when the signal arrives at a very low (less than $10^{\circ}$ ) or negative elevation angle (surface glance), it can cause complete signal loss.

These effects can be represented, for code(1) and carrier(2), in simplified form as:

$$
\begin{gathered}
R=R_{R T}+\delta_{M}+\varepsilon \\
\Phi=\frac{c}{\lambda}\left(\Delta \delta+\delta_{M}\right)+N+\varepsilon
\end{gathered}
$$

Where $R$ indicates pseudorange (code), $\Phi$ carrier phase, $c$ the speed of light constant, $\lambda$ wavelength, $\mathrm{N}$ ambiguity, $\delta_{M}$ multipath and $\varepsilon$ remaining noise. Generally, phase multipath effects tend to be smaller than $\frac{1}{4}$ of a phase (Yang et al. 2010), causing a bias up to $3 \mathrm{~cm}$ for Locata.

The Locata system introduces a number of noise mitigating techniques, such as dual frequency and spatially separated antennas (for the single transmitter) (Barnes et al. 2006).

A default threshold of $\frac{1}{3}$ of the phase indicates that multipath remains undetected.

One of previously suggested correction methods is to treat multipath as a systematic error (Yang et al. 2010) and remove it accordingly. Authors are assessing how external factors, such as lowering the transmission level and introducing dedicated antenna shielding can improve indoor performance.

\subsection{Datasets}

Datasets has been collected in 3 different locations:

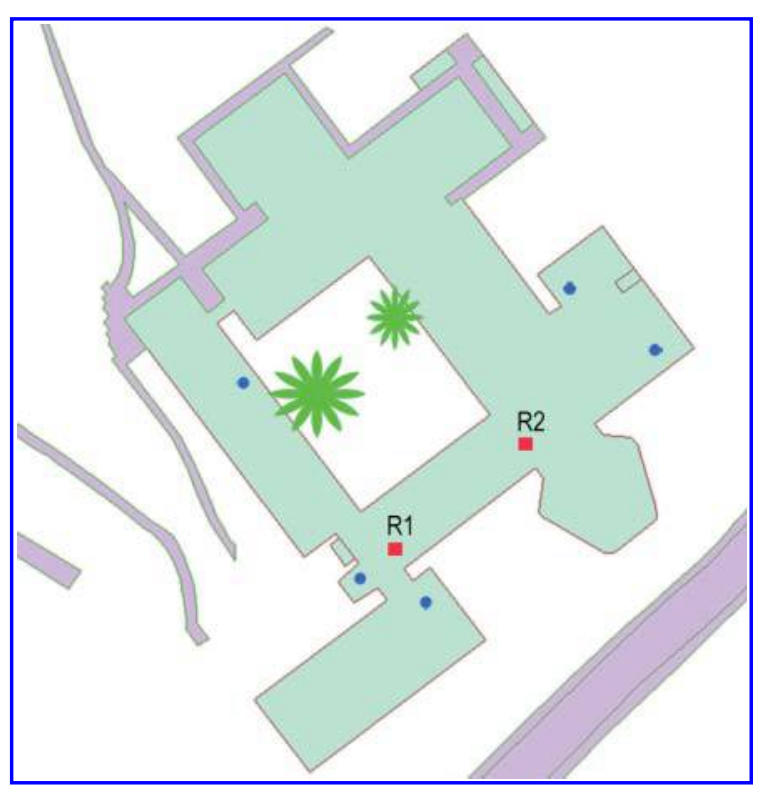

Figure 2: Environment B Dataset. 
A. Locata's test network in Australia, with network edges exceeding $2 \mathrm{~km}$ and height differences up to $150 \mathrm{~m}$.

B. Small network at the University Park (University of Nottingham). Several obstructions, including high trees and buildings were present. Locata transreceivers (blue dots on the Figure 2) are nearly coplanar.

C. Data collected inside the new Nottingham Geospatial Building at the Jubilee Campus of the University of Nottingham (Figure 3).

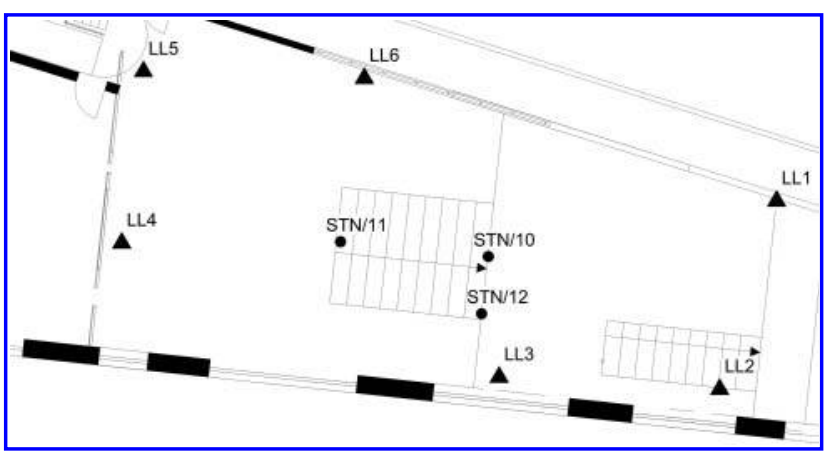

Figure 3: Enviroment C Network.

The research focuses on the dual frequency Locata system's capacity to mitigate noise in the indoor environment $(\mathrm{C})$, with outdoor tests $(\mathrm{A} \& \mathrm{~B})$ used as a comparison.

\subsection{Description of test methods}

Most of the presented results have been calculated within the receiver provided by Locata. Data has also been post processed using Locata's proprietary software (LINE). The following scenarios have been used for post processing:

- H0 uses all the signals

- $\mathrm{H} 2$ forms one measurement by averaging 4 signal clusters

- H3 forms one measurement by averaging 4 signal clusters, weighted by signal to noise ratio (SNR)

All scenarios utilise the same settings as rover, so multipath may still remain undetected (as per 2.1). Certain datasets, analysed in Section 3, not solvable in real time by the rover have been postprocessed using filtered data (by removing a section of very noisy data at the start). On this occasion $\mathrm{H} 2$ scenario was used, as it is the most similar to receiver's real-time solution.
Receiver also outputs DOP values and the Locata's proprietary Signal to Noise Ratio (SNR), described sometimes as Locata Signal Strength Indicator (LSSI). The authors' software was used to extract the Low Correlation Output Events (LCOE) parameter. It is a count of events, within each epoch, when statistical tests indicate that correlator output should be discarded, usually indicating interference (Khan et al. 2010).

The statistical a priori and a posteriori approach assesses datasets by calculating 2D error ellipses. Semi major and minor axes $(a, b)$ and azimuth $(\alpha)$ are calculated from the symmetric covariance matrix $Q$ :

$$
\begin{aligned}
Q & =\left[\begin{array}{ll}
\partial_{x}^{2} & \partial_{x y} \\
& \partial_{y}^{2}
\end{array}\right] \\
a & =\sqrt{\lambda_{\max }} \\
& =\sqrt{\frac{1}{2}\left(\partial_{x}^{2}+\partial_{y}^{2}+\sqrt{\partial_{x}^{2}-\partial_{y}^{2}+4\left(\partial_{x y}\right)^{2}}\right)} \\
b & =\sqrt{\lambda_{\min }} \\
& =\sqrt{\frac{1}{2}\left(\partial_{x}^{2}+\partial_{y}^{2}-\sqrt{\partial_{x}^{2}-\partial_{y}^{2}+4\left(\partial_{x y}\right)^{2}}\right)} \\
\tan \alpha & =\frac{\partial_{x y}}{a^{2}-\partial_{x}^{2}} .
\end{aligned}
$$

A priori covariance $Q$ was based on the least squares minimal constrained solution (one point and the azimuth fixed), with Locata represented as a cm accuracy distance measurements.

A posteriori values were calculated from the collected data. Generally, those results should be similar, unless an external factor has been present.

By-products of the a posteriori calculations were precision $\left(\mathrm{SD}_{\mathrm{X}}\right)$ and accuracy $\left(\mathrm{d}_{\mathrm{X}}\right)$, if the true value is known (by independent GPS or tachymetric measurements). On such occasions the distance from the true value was marked as $\left(\mathrm{d}_{\mathrm{D}}\right)$. All statistical values are given at $95 \%$ confidence level.

\subsection{Scenarios comparison}

Unless stated otherwise all presented data has been collected in static mode. At present Locata is not solving ambiguities on-the-fly (OTF) but initialisation occurs on a on the known point instead. Given that the constant part of noise (multipath) is being mostly absorbed by the initialisation method, the observed results do not present absolute noise, but mostly its fluctuation due to the environmental changes. Additional observations, such as fix rate, are used to assess this effect more fully. 
Table 1: A priori and a posterior error ellipses comparison.

\begin{tabular}{|c|c|c|c|c|c|c|c|c|}
\hline \multirow{2}{*}{$\begin{array}{l}95 \% \\
\text { confi- } \\
\text { dence } \\
\text { level }\end{array}$} & \multicolumn{3}{|c|}{ a priori } & \multicolumn{3}{|c|}{ a posterior } & \multirow[t]{2}{*}{ HDOP } & \multirow{2}{*}{$\begin{array}{l}\text { No. of } \\
\text { signals }\end{array}$} \\
\hline & $\mathrm{a}[\mathrm{m}]$ & $\mathrm{b}[\mathrm{m}]$ & $\alpha\left[^{0}\right]$ & $\mathrm{a}[\mathrm{m}]$ & $\mathrm{B}[\mathrm{m}]$ & $\alpha\left[^{o}\right]$ & & \\
\hline A & 0.020 & 0.009 & 108 & 0.006 & 0.005 & 117 & 0.8 & 39 \\
\hline B & 0.024 & 0.012 & 134 & 0.031 & 0.006 & 127 & 1.6 & 20 \\
\hline $\mathrm{C}$ & 0.022 & 0.013 & 27 & 0.049 & 0.015 & 180 & 1.2 & 24 \\
\hline
\end{tabular}

Despite those restrictions, comparison of datasets (described in 2.2), show decorrelation between HDOP and ellipse shape distortion $<3 \mathrm{~cm}$ (Table $1)$. This suggest multipath and noise prevailing effects.

\section{Nottingham Geospatial Building Indoor Tests}

The comparison of results from Table 1 shows that noise (multipath) is especially prominent indoors (C). This data was collected in the newly constructed and actively occupied Nottingham Geospatial Building (NGB). Locata transmitter placements (Figure 4) were chosen to minimise disturbance and create the optimal possible geometry. Figure 3 presents the experiment area (roughly $25 \times 10 \mathrm{~m}$ ) with transceivers marked as triangles and the receivers as dots. Three locations (STN/10-STN/12) were selected to reduce any biases in the experiment.

The tests lasted for a week, and 32 sets of static carrier phase observations have been collected, by alternating one or more of following factors:

- linear gain for the antenna and firmware settings

- noise shielding mechanism

- point location (STN/10-12)

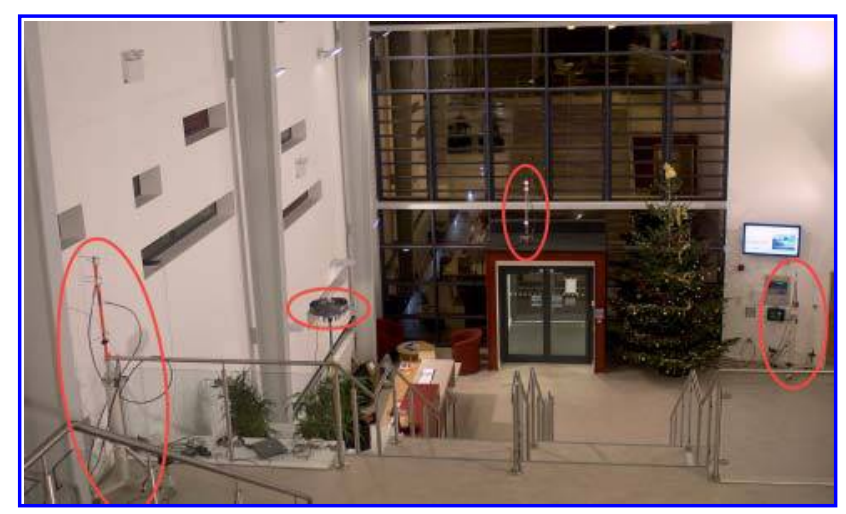

Figure 4: Locata inside the NGB.
As explained in 2.3 certain datasets could only be solved by postprocessing filtered data (by removing a section of very noisy data at the start of data collection).

The accuracy threshold criteria $\left(\mathrm{d}_{\mathrm{D}}<0.04 \mathrm{~m}\right)$ (Tables 2-4) was established based on the multipath and cycle slip detection characteristics (as explained in 2.1). Out of 32, 24 sets passed the criteria and were further processed. Results in 3.2-3.3 are an average of measurement sets falling into specific category.

As a validation of those results, two specific datasets (Set I \& II) have been selected and discussed in 3.4.

\subsection{Interference}

Extensive lab tests (Khan et al. 2010) indicated that other devices using the ISM band might interfere with the Locata signal and therefore have a detrimental effect on the position quality of the system. Most notorious are Wi-Fi hotspots, which are very common in urban environments.

The authors' measurements, by dedicated software (Figure 5), show a number of WiFi signals overlapping Locata's S1\&S6 frequencies, with strength $-60 \mathrm{dBm}$ or lower, weak compared with Locata $(2-20 \mathrm{dBm})$.

LOCE (Figure 6) from Set I \& II (3.4) is notably larger in S6 frequency, more susceptible to Wi-Fi interference. It does not though follow the pattern de-

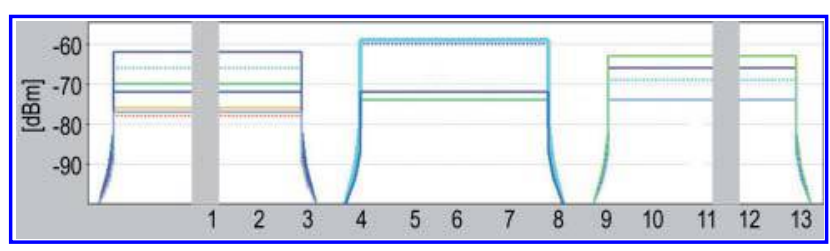

Figure 5: WiFi signal inside NGB with S1\&S6 indicated in grey.

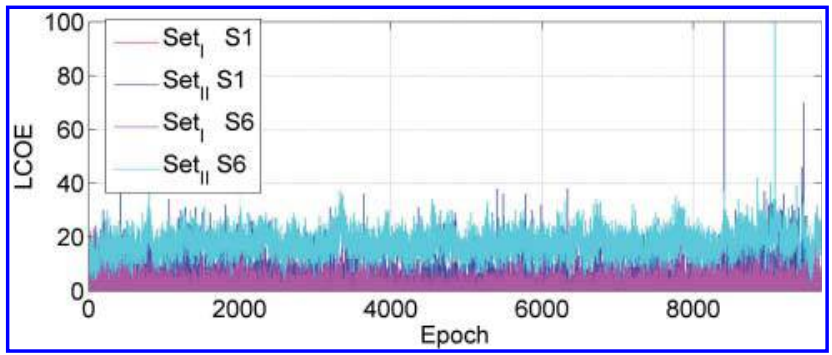

Figure 6: LCOE for Sets I \& II 
Table 2: Effect of Linear Gain (LGA) on position accuracy.

\begin{tabular}{cllllll}
\hline $\begin{array}{l}\mathrm{LGA} \\
{[\mathrm{dBm}]}\end{array}$ & $\begin{array}{l}\mathrm{SD}_{\mathrm{E}} \\
{[\mathrm{m}]}\end{array}$ & $\begin{array}{l}\mathrm{SD}_{\mathrm{N}} \\
{[\mathrm{m}]}\end{array}$ & \multicolumn{1}{l}{$\begin{array}{l}\mathrm{d}_{\mathrm{E}} \\
{[\mathrm{m}]}\end{array}$} & $\begin{array}{l}\mathrm{d}_{\mathrm{N}} \\
{[\mathrm{m}]}\end{array}$ & $\begin{array}{l}\mathrm{d}_{\mathrm{D}} \\
{[\mathrm{m}]}\end{array}$ & $\%$ Fix \\
\hline+20 & 0.013 & 0.021 & 0.004 & -0.001 & 0.004 & 52.2 \\
+8 & 0.008 & 0.014 & -0.005 & -0.005 & 0.007 & 89.9 \\
+2 & 0.011 & 0.009 & -0.001 & -0.002 & 0.002 & 90.8 \\
-4 & 0.004 & 0.009 & 0.000 & -0.001 & 0.001 & 99.9 \\
\hline
\end{tabular}

scribed in (Khan et al. 2010), and is more prominent with Set II, which yield better results (3.4).

This leads to the conclusion that while Wi-fi hotspots interfere with the Locata signal, it doesn't seem to cause visible problem in the real life situations, when hotspots are unlikely to be located next to Locata transmitters or receivers. A more in depth study of the effects of Wi-Fi to the Locata system in an indoor environment, would be recommended to fully assess this.

\subsection{Alteration of Linear Gain}

Lowering the power of transmission (LGA) showed improvement in accuracy $\left(\mathrm{SD}_{\mathrm{X}}\right)$ and Fix Ratio (Table 2). Precision $\left(\mathrm{d}_{\mathrm{X}}\right)$ remained below expected multipath value, but the pattern is only visible between extremes $(+20 \mathrm{dBm}$ and $-4 \mathrm{dBm})$.

Precision decrease for $+8 \mathrm{dbm}$ values could be caused by standing multipath affecting one or more transmitters. This can cause suboptimal performance of TimeLoc, introducing transmitter clock error. Although small, this effect could be eliminated by close monitoring of initial setup.

\subsection{Shielding mechanism}

Antenna used by the Locata rover has no cut off angle and is sensitive to all incoming signal. It is generally assumed that the most prominent multipath tends to be created in the direct vicinity of the receiver. Authors decided to verify if shielding (Figure 7) could mitigate this problem. Three scenarios have been compared:

- Antenna with no shielding, treated as baseline.

- Antenna shielded by a small metal plate $(0.30 \mathrm{~m}$ dia), intended to eliminated low angle multipath.

- Antenna shielded by choke ring antenna (CR), designed for longer GNSS wavelength, but in theory capable of eliminating most of Locata multipath.

Shielding proves to be successful, with results signifying low angle multipath as a prominent noise. Comparison between precision and accuracy indi-

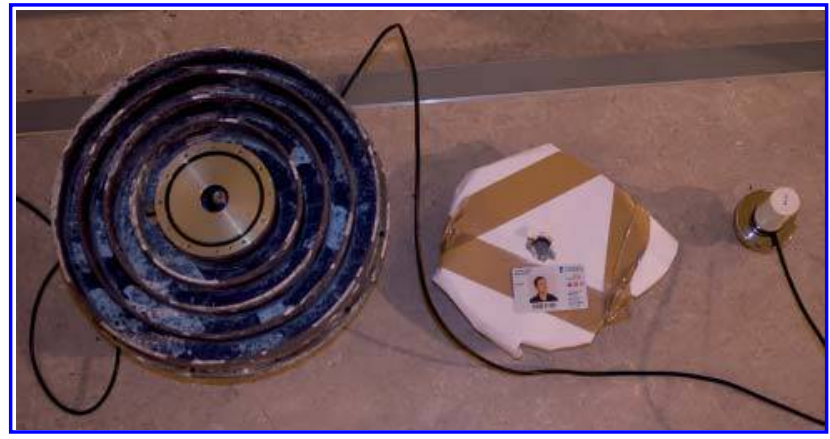

Figure 7: Shielding - choke ring, iron plate and Locata antenna.

Table 3: Effect of the shielding mechanism on the position accuracy.

\begin{tabular}{lllllll}
\hline Type & $\begin{array}{l}\mathrm{SD}_{\mathrm{E}} \\
{[\mathrm{m}]}\end{array}$ & $\begin{array}{l}\mathrm{SD}_{\mathrm{N}} \\
{[\mathrm{m}]}\end{array}$ & $\begin{array}{l}\mathrm{d}_{\mathrm{E}} \\
{[\mathrm{m}]}\end{array}$ & \multicolumn{1}{l}{$\begin{array}{l}\mathrm{d}_{\mathrm{N}} \\
{[\mathrm{m}]}\end{array}$} & \multicolumn{1}{l}{$\begin{array}{l}\mathrm{d}_{\mathrm{D}} \\
{[\mathrm{m}]}\end{array}$} & $\%$ Fix \\
\hline Nothing & 0.003 & 0.011 & 0.000 & 0.011 & 0.007 & 66.3 \\
Plate & 0.010 & 0.016 & 0.004 & -0.010 & 0.004 & 78.9 \\
CR & 0.007 & 0.005 & 0.003 & -0.005 & 0.004 & 98.2 \\
\hline
\end{tabular}

cates that no bias is present, thought plate's $\mathrm{SD}_{\mathrm{X}}$ suggest that small multipath might have been introduced.

\subsection{Case study}

In order to verify findings from Sections 3.2 and 3.3, two observation sets, measured on same point (STN/10), has been selected:

- Set $\mathrm{I}-$ a high power setting $(+20 \mathrm{dBm})$ with plate shielding.

- Set II - low power setting $(+2 \mathrm{dBm})$ with a choke ring.

In Table 4 OL stands for online results and $\mathrm{H} 0$ and $\mathrm{H} 3$ respectively for direct and weighted postpocessing mode (see 2.3). Both sets had almost $100 \%$ fix rate, though the data from Set I was filtered by removing the noisy data at the start of the measurements, as explained in 2.3.

Set II H0 scenario matches a priori assessment (a, b, $\alpha$ ) with postprocess and OL increasing accuracy and shifting minor and major axis of error ellipses.

Set I H0 seems to be affected by a bias, which cannot be removed fully by postprocessing. OL and $\mathrm{H} 3$ results are inconsistent, suggesting that collected data is of poorer quality. 
Table 4: Postprocessing comparison.

\begin{tabular}{|c|c|c|c|c|c|c|c|}
\hline \multicolumn{2}{|c|}{ Set } & $\begin{array}{l}\mathrm{SD}_{\mathrm{E}} \\
{[\mathrm{m}]}\end{array}$ & $\begin{array}{l}\mathrm{SD}_{\mathrm{N}} \\
{[\mathrm{m}]}\end{array}$ & $\begin{array}{l}\mathrm{d}_{\mathrm{E}} \\
{[\mathrm{m}]}\end{array}$ & $\begin{array}{l}\mathrm{d}_{\mathrm{N}} \\
{[\mathrm{m}]}\end{array}$ & $\begin{array}{l}\mathrm{d}_{\mathrm{D}} \\
{[\mathrm{m}]}\end{array}$ & $\begin{array}{l}\alpha \\
{\left[{ }^{\circ}\right]}\end{array}$ \\
\hline \multirow[t]{3}{*}{ I } & $\mathrm{OL}$ & 0.009 & 0.013 & 0.006 & 0.010 & 0.006 & 33.4 \\
\hline & H0 & 0.009 & 0.005 & 0.002 & -0.004 & 0.002 & 113.5 \\
\hline & H3 & 0.012 & 0.011 & 0.003 & 0.002 & 0.003 & 46.5 \\
\hline \multirow[t]{3}{*}{ II } & OL & 0.009 & 0.006 & 0.0 & 0.0 & 0.0 & 118.7 \\
\hline & $\mathrm{H} 0$ & 0.015 & 0.022 & 0.025 & -0.006 & 0.025 & 24.3 \\
\hline & $\mathrm{H} 3$ & 0.009 & 0.005 & 0.006 & 0.001 & 0.006 & 118.5 \\
\hline \multicolumn{4}{|c|}{ a priori error ellipse } & 0.023 & 0.013 & & 26.7 \\
\hline
\end{tabular}

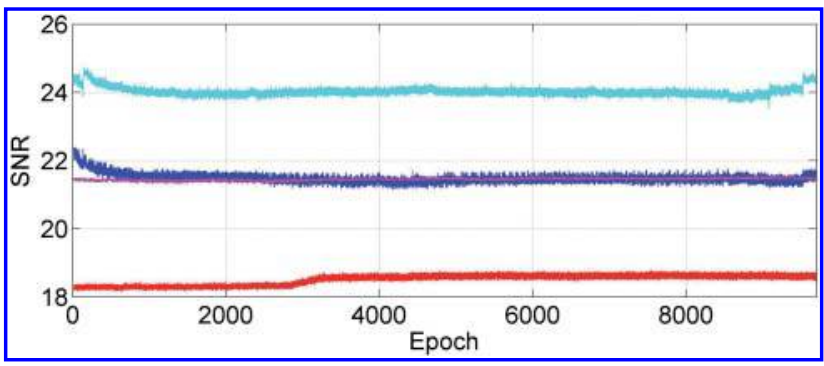

Figure 8: SNR/LSSI comparison between Set I \& II (Figure 6 colour scheme).

Generally the results agree with previous ones (3.2, 3.3) and SNR analysis (Figure 8). Performance of Set II (magenta and blue) is visibly better. Also S6 performance is better, probably due to lower interference (Figure 6). Changing SNR values indicate variation in environmental conditions of the tests.

\section{Conclusion}

This paper has presented the results of experiments and shown Locata capacity of centimetre level static positioning in urban and indoor environments. By comparing different power levels and shielding options (metal plate and a choke ring) authors investigated its efficiency in mitigating the noise (multipath) in an indoor environment. Results suggest certain success in this approach. Choke ring and low power settings seemed to remove most of multipath effect. Shielding low angle signal is not a problem indoors where most Locatalites are expected to be placed higher than rover.

The lack of on the fly (OTF) ambiguity resolution is obviously one of the shortcomings of Locata system. Some work in this area, ambiguities calculation using LAMBDA method, is described in (Bertsch et al. 2009). Another approach is integrated system, as discussed below.

\subsection{Future work}

GNSS ease of use makes it favourite for monitoring of manmade structures and machine guidance. Locata has already been tested for those applications (Barnes et al. 2007) in areas of low sky visibility.

Reasonably high frequency measurements are required for monitoring applications of structures such as bridges or buildings. GPS measurements of at least $10 \mathrm{~Hz}$ are used to detect natural frequencies of the larger structures (Ogundipe et al. 2008). A combination of high frequency GNSS receivers $(20 \mathrm{~Hz})$ and total stations (Brown et al. 2007) provide a monitoring for wind load of high rise structures. Locata is capable of measurements up to $50 \mathrm{~Hz}$ with sub centimetre accuracy (Bonenberg et al. 2009).

While Locata gives the advantage of network design flexibility, on its own it struggles with height determination. But an integrated system brings advantage of strong terrestrial signal $(23 \mathrm{dBm}$ versus $-130 \mathrm{dBm}$ ), with no near-far effect, improved height determination and integrity control. Authors are expecting that tight integration GPS and Locata will deliver the following:

- Partly mitigate the requirement of the sky visibility.

- Maintain centimetre level accuracy in most environmental conditions.

- Monitor accuracy and system integrity.

- Benefit from enhanced geometry.

\section{Acknowledgement}

This research is supported by the Engineering and Physical Sciences Research Council.

Authors would like to extend gratitude to all people involved in the work on this paper. Dr. Joel Barnes from Locata Corporation and Nonie Politi from University of New South Wales provided valuable support and data. Also, the support from Mr Huib de Ligt and Dr. Oluropo Ogundipe from IESSG has been very important.

\section{References}

Barnes, J., van Cranenbroeck, J., Rizos, C., Pahwa, A. and Politi, N., Long term performance analysis of a new ground-transceiver positioning network (LocataNet) for structural deformation monitoring applications, 2007. 
Barnes, J., Rizos, C., Kanli, M., Pahwa, A. and Politi, N., Locata: A new positioning technology for classically difficult GNSS environments, Proceedings of the 2006 IGNSS Symposium.

Bertsch, J., Choudhury, M., Rozos, C. and Kahle, H. G., On-the-Fly Ambiguity Resolution for Locata, Proceedings of the 2009 IGNSS Symposium, Australia, December 2009.

Bonenberg, L. K., Roberts, G. W., Hancock, C. M., Ogundipe, O. and Lee, J. K., Feasibility of integrated Locata and GNSS for Engineering Work Application, Proceedings of the 2009 International Symposium on GPS/GNSS, Korea, November 2009.

Brown, N., Kaloustian, S. and Roeckle, M., Monitoring of Open Pit Mines using Combined GNSS Satellite Receivers and Robotic Total Stations, 2007 Inernational Symposium on Rock Slope Stability, 12-14 September 2007.

Khan, F., Rizos, C. and Dempster, A., Locata Performance Evaluation in the Presence of Wide and Narrow-Band Interference, 2010.

Lee, H. K., Soon, B., Barnes, J., Wang, J. and Rizos, C., Experimental Analysis of GPS/Pseudolite/INS Integration for Aircraft Precision Approach and Landing, The Journal Of Navigation (2008), 61, pp. 257-270.

Leick, A., GPS Satellite Surveying, Third Edition, Wiley, 2004.

Meng, X., Roberts, G. W., Dodson, A. H., Cosser, E., Barnes, J. and Rizos, C., Impact of GPS satellite and pseudolite geometry on structural deformation monitoring: analytical and empirical studies, Journal of Geodesy (2003), 77: 809-822.

Montillet, J. P., Roberts, G. W., Meng, X., Taha, A., Hancock, C., Ogundipe, O. and Barnes, J., Deploying a Locata network to enable precise positioning in urban canyons. J Geod (2009), 83:91-103.

Ogundipe, O., Roberts, G. W. and Brown, C., Identifying The Frequencies of a Twin Steel Box Girder Viaduct By GPS Monitoring, Proceedings of the 2008 International Symposium on GPS/ GNSS, Tokyo (2008).
Roberts, G. W., Meng, X., Brown, C. J. and Dallard, P., GPS measurements on the London Millennium Bridge, Proceedings of the Institution of Civil Engineers, Bridge Engineering 159, December 2006, Issue BE4, pp. 153-161.

Yang, G., He, X. and Chen, Y., Integrated GPS and Pseudolite positioning for deformation monitoring, Survey Review, 42, 315 pp. 72-81, January 2010.

Received: Jan 23, 2010 Accepted: Aug 12, 2010

\section{Author information}

Lukasz Kosma Bonenberg, Craig M. Hancock and

Gethin W. Roberts

IESSG

The University of Nottingham

The Nottingham Geospatial Building

Triumph Road

Nottingham NG7 2TU, United Kingdom

E-mail: isxlkb@nottingham.ac.uk 